\title{
Article \\ Analysis of the Applicability of microRNAs in Peripheral Blood Leukocytes as Biomarkers of Sensitivity and Exposure to Fractionated Radiotherapy towards Breast Cancer
}

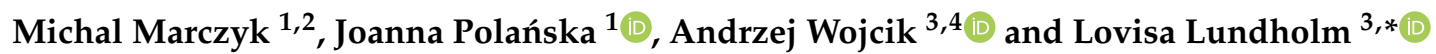 \\ 1 Department of Data Science and Engineering, Silesian University of Technology, 44-100 Gliwice, Poland; \\ michal.marczyk@polsl.pl (M.M.); joanna.polanska@polsl.pl (J.P.) \\ 2 Yale Cancer Center, Yale School of Medicine, New Haven, CT 06511, USA \\ 3 Department of Molecular Biosciences, The Wenner-Gren Institute, Stockholm University, \\ 10691 Stockholm, Sweden; andrzej.wojcik@su.se \\ 4 Institute of Biology, Jan Kochanowski University, 25-406 Kielce, Poland \\ * Correspondence: lovisa.lundholm@su.se
}

check for updates

Citation: Marczyk, M.; Polańska, J.; Wojcik, A.; Lundholm, L. Analysis of the Applicability of microRNAs in Peripheral Blood Leukocytes as Biomarkers of Sensitivity and Exposure to Fractionated Radiotherapy towards Breast Cancer. Int. J. Mol. Sci. 2021, 22, 8705.

https://doi.org/10.3390/ijms22168705

Academic Editor: Orla L. Howe

Received: 30 June 2021

Accepted: 10 August 2021

Published: 13 August 2021

Publisher's Note: MDPI stays neutral with regard to jurisdictional claims in published maps and institutional affiliations.

Copyright: (c) 2021 by the authors. Licensee MDPI, Basel, Switzerland. This article is an open access article distributed under the terms and conditions of the Creative Commons Attribution (CC BY) license (https:// creativecommons.org/licenses/by/ $4.0 /)$.

\begin{abstract}
Biomarkers for predicting individual response to radiation and for dose verification are needed to improve radiotherapy. A biomarker should optimally show signal fidelity, meaning that its level is stable and proportional to the absorbed dose. miRNA levels in human blood serum were suggested as promising biomarkers. The aim of the present investigation was to test the miRNA biomarker in leukocytes of breast cancer patients undergoing external beam radiotherapy. Leukocytes were isolated from blood samples collected prior to exposure (control); on the day when a total dose of $2 \mathrm{~Gy}, 10 \mathrm{~Gy}$, or $20 \mathrm{~Gy}$ was reached; and one month after therapy ended (46-50 Gy in total). RNA sequencing was performed and univariate analysis was used to analyse the effect of the radiation dose on the expression of single miRNAs. To check if combinations of miRNAs can predict absorbed dose, a multinomial logistic regression model was built using a training set from eight patients (representing 40 samples) and a validation set with samples from the remaining eight patients (15 samples). Finally, Broadside, an explorative interaction mining tool, was used to extract sets of interacting miRNAs. The most prominently increased miRNA was miR-744-5p, followed by miR-4461, miR-34a-5p, miR-6513-5p, miR-1246, and miR-454-3p. Decreased miRNAs were miR-3065-3p, miR-103a-2-5p, miR-30b-3p, and miR-5690. Generally, most miRNAs showed a relatively strong inter-individual variability and different temporal patterns over the course of radiotherapy. In conclusion, miR-744-5p shows promise as a stable miRNA marker, but most tested miRNAs displayed individual signal variability which, at least in this setting, may exclude them as sensitive biomarkers of radiation response.
\end{abstract}

Keywords: miRNA; radiation; biomarker; radiotherapy

\section{Introduction}

Radiation therapy is an extreme form of planned radiation exposure in that it involves very high doses which are lethal if given to a large part of the body [1]. Patients survive radiotherapy because the high dose is confined to the tumour tissue and exposure of normal tissue is minimised. Nevertheless, radiotherapy is associated with risks of side effects, both of deterministic (tissue damage) [2] and stochastic (cancer) nature [3].

A certain degree of early and late tissue reactions is an inherent element of radiotherapy. This is because the radiation doses given to critical organs are on the upper edge of tolerability, which is defined for an average patient [4]. Nevertheless, some patients develop severe side effects and one of the components may be a genetic-based, individual sensitivity to radiation of normal tissues [5,6]. Attempts are being undertaken to develop biomarkers of individual sensitivity to radiation so that highly sensitive patients can be 
identified prior to therapy [7,8]. Additionally, radiotherapy is a double-edged sword in its use as a cancer treatment-being itself a carcinogen. Not surprisingly, patients who are cured by radiotherapy live with an elevated risk of suffering from radiation-induced cancers, which are termed second cancers or second malignant neoplasms (SMN) [3]. Biomarkers of susceptibility to radiation-induced damage might also help in identifying patients with an enhanced risk of second cancers [8].

Given the fact that doses absorbed by normal tissues in the course of radiotherapy are on the upper edge of their tolerability, precision of dose delivery and quality control are elementary to the procedure [9]. Accidental over- and underexposures do occur and have deleterious effects [1]. Overexposures lead to development of life-threatening normal tissue reactions, and underexposures to loss of tumour control. Application of dose verification methods is recommended, but the classical in vivo dosimetry using physical dosimeters is increasingly difficult due to the development of sophisticated RT techniques $[10,11]$. An alternative in vivo dosimetry method is based on analysing biomarkers of radiation exposure. Here, the gold standard is the dicentric test for peripheral blood lymphocytes. Its applicability for verifying the doses absorbed during radiotherapy was tested by several authors and its general problem was found to be the high inter-individual variability (summarised in [12]).

Whether a biomarker is used for assessing the individual response to radiation or for estimating the absorbed dose, an important criterion determining its usability is signal fidelity. By this we mean that its level is stable upon repeated sample collection and that it shows a consistent relationship to the absorbed dose. miRNA levels in mouse blood serum have been suggested as promising biomarkers of absorbed dose $[13,14]$. The aim of the present investigation was to test the fidelity of leukocyte miRNA expression as an in vivo dosimeter. To this end we collected peripheral blood mononuclear cells (PBMC) from patients before, during, and after external radiotherapy for breast cancer. Global levels of miRNA were measured by RNA sequencing. miRNA candidates were selected by univariate analysis, multinomial logistic regression model building, and interaction searches.

\section{Results}

\subsection{Sample Description}

Blood was sampled from breast cancer patients given fractionated radiotherapy, where each patient received 2 Gy per fraction, five days per week (Monday-Friday). Sampling was performed before radiotherapy, on the day of exposure to 2, $10(5 \times 2)$, and $20(10 \times 2) \mathrm{Gy}$, and one month after the full fractionation scheme ended, which varied between 23 and 25 fractions of 2 Gy (" 1 month", for 46 or 50 Gy in total from 23 or $25 \times 2$ Gy, respectively, is used throughout the figures/tables to discriminate the late from the earlier responses). Of the original cohort of 16 breast cancer patients, due to technical reasons (see Patient Samples section) we had complete sets of samples from only five patients (patients 7, 9, 14-16) and all doses, except one, for three patients (patients 8, 12,13) (Table 1). This eight-patient set, representing 40 samples after applying imputation of missing dose measurements (see Section 4.4), was combined into a training set, while the remaining patient specimens were used as a validation set (15 samples). 
Table 1. Description of patients and samples used in the study. The group column shows which patient sample sets were used in the training or validation cohort. The total dose column shows the doses (given in 2 Gy fractions over five days per week) received by the patients after which miRNA expression could (green colour) or could not (no colour) be measured. Sampling was performed on the day when an accumulated dose of 2, 10, and 20 Gy, and one (1) month after the full dose of 46 or $50 \mathrm{~Gy}$, was reached.

\begin{tabular}{|c|c|c|c|c|c|c|c|c|}
\hline \multirow{2}{*}{ Patient ID } & \multirow{2}{*}{ Age } & \multirow{2}{*}{ Smoker } & \multirow{2}{*}{ Group } & \multicolumn{5}{|c|}{ Total Dose [Gy] } \\
\hline & & & & 0 & 2 & 10 & 20 & 46-50/1 Month \\
\hline 1 & 61 & No & Validation & & & & & \\
\hline 2 & 65 & No & Validation & & & & & \\
\hline 3 & 56 & No & Validation & & & & & \\
\hline 4 & 72 & No & Validation & & & & & \\
\hline 5 & 74 & No & Validation & & & & & \\
\hline 6 & 66 & NK & Validation & & & & & \\
\hline 7 & 57 & Yes & Training & & & & & \\
\hline 8 & 67 & No & Training & & & & & \\
\hline 9 & 64 & No & Training & & & & & \\
\hline 10 & 75 & No & Validation & & & & & \\
\hline 11 & 81 & No & Validation & & & & & \\
\hline 12 & 65 & No & Training & & & & & \\
\hline 13 & 58 & Yes & Training & & & & & \\
\hline 14 & 65 & No & Training & & & & & \\
\hline 15 & 68 & Yes & Training & & & & & \\
\hline 16 & 60 & No & Training & & & & & \\
\hline
\end{tabular}

\subsection{Principal Component Analysis}

We first analysed whether blood samples collected after the various doses could be differentiated. Sequencing libraries from all samples were of similar quality. The outlier and missing expression values were randomly spread across different miRNAs and samples (Figure S1). After normalization, miRNA expression measurements were comparable between all samples (Figure S2). Principal component analysis (PCA) based on 573 miRNAs left after removing low expression data (see Section 4.4), shows that the different doses received by the patients could not be discriminated from one another (Figure 1).
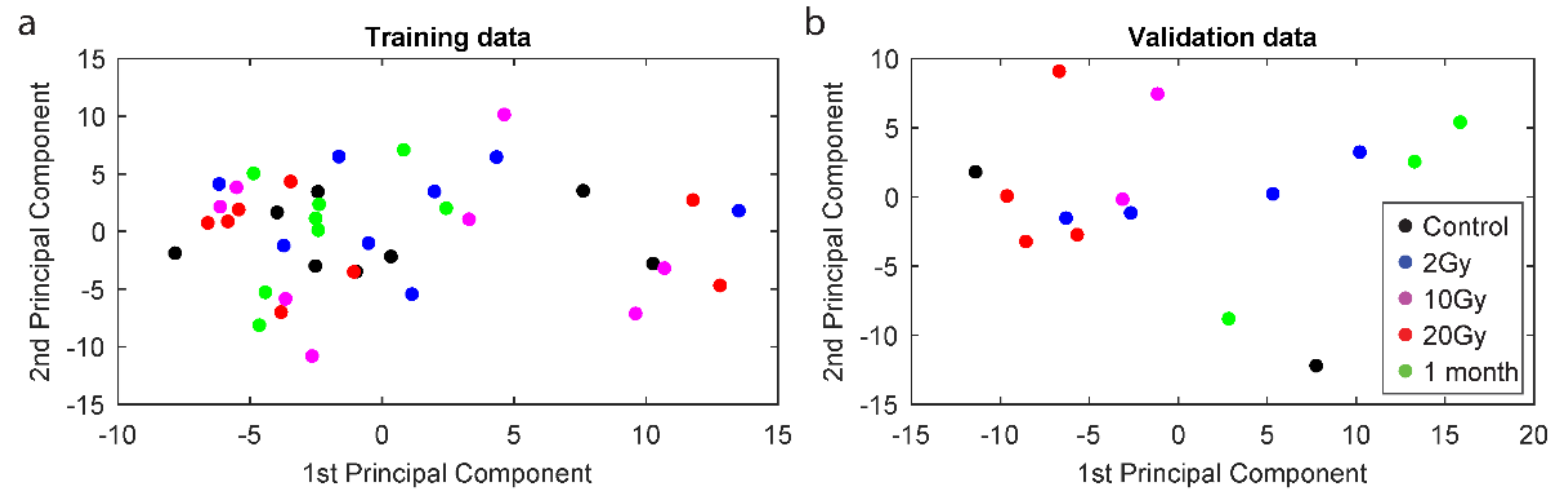

Figure 1. Principal component analysis plot based on 573 miRNAs left after filtering with each dose/collection time point in a different colour. The accumulated dose is shown in all figures, where $10 \mathrm{~Gy}$ was given as $5 \times 2 \mathrm{~Gy}, 20 \mathrm{~Gy}$ as $10 \times 2 \mathrm{~Gy}$, and 1 month represents the time of sampling after reaching, in total, $46-50 \mathrm{~Gy}$, given as 23 or $25 \times 2$ Gy. Euclidean distance was used. Training sets (a) and validation sets (b) were analysed separately.

\subsection{Radiation-Responsive miRNAs}

Next, we analysed the trends of radiation-induced miRNA changes in the training set by pairwise comparison between each dose. Two hundred and fifty-six miRNAs 
were differentially expressed (DE) in at least one comparison (uncorrected $p$-value $<0.05$ ). Overall, there were more up- than downregulated miRNAs versus the control or the adjacent dose (Table 2). The highest number of DE miRNA was observed in the 2 vs. 0 Gy comparison, which reflects a fast response to radiation, and at 1 month after therapy ended vs. $0 \mathrm{~Gy}$, which reflects a late response (Figure 2a). Most DE miRNAs were unique to each dose comparison. When false discovery rate (FDR) control was applied, only miR-3065-3p was significantly downregulated at $2 \mathrm{~Gy}$ in comparison to control, with the same trend after the other doses. However, a large inter-individual variability was observed (Figure 2b).
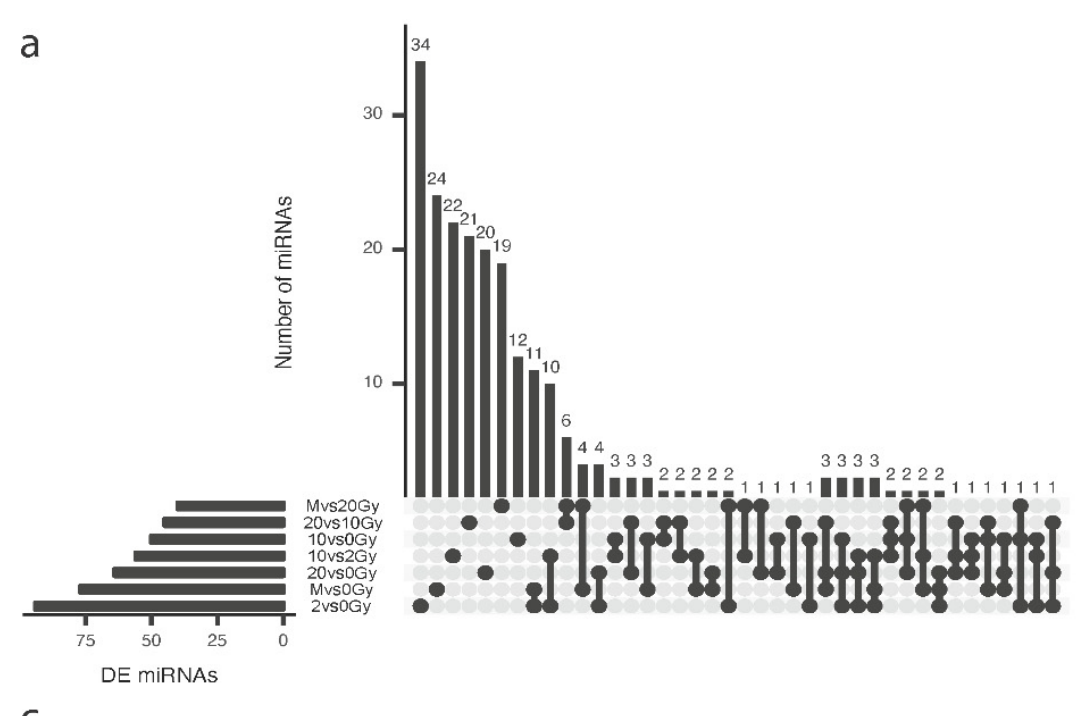

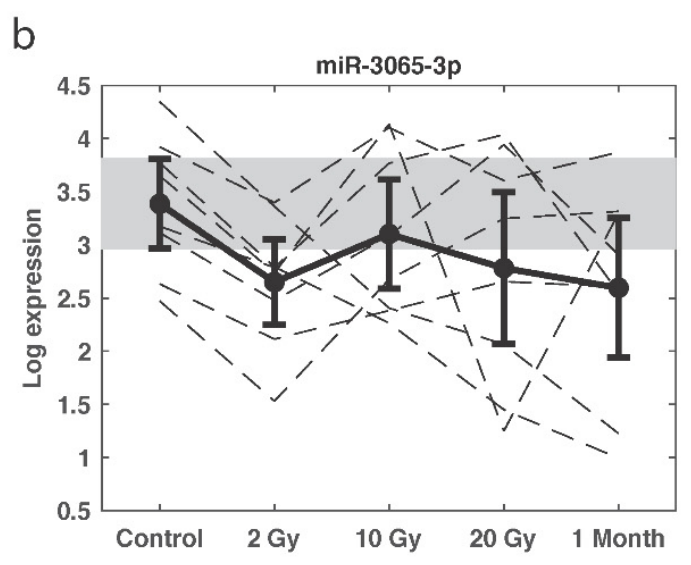

C

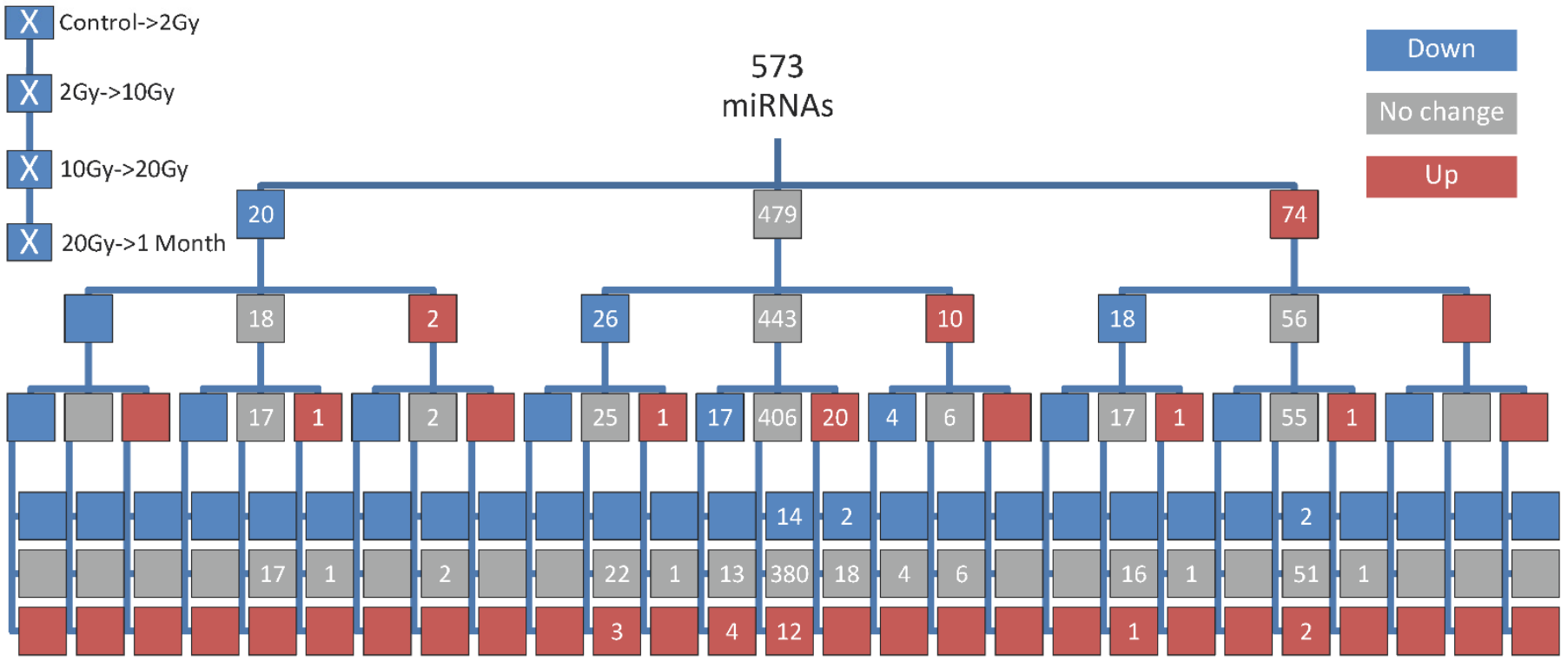

Figure 2. Discovery of differentially expressed (DE) miRNAs analysed in the training cohort. (a) UpSet plot that shows intersection of DE miRNAs among comparisons between different total doses. M represents one month after end of therapy (46/50 Gy in total); (b) Expression of a significantly different miRNA in 2 Gy versus control, after multiple testing correction; (c) Trend patterns diagram of miRNAs. Box colours represent downregulation (blue), no change (grey), or upregulation (red) versus the adjacent, lower total dose (or versus control for $2 \mathrm{~Gy}$ ). The lowest branch level is drawn vertically to save space. The numerical value corresponds to the number of miRNAs in that branch. 
Table 2. Number of regulated miRNAs in the training set when comparing two groups using a t-test, prior to multiple correction. The numbers $0,2,10$, and 20 represent total doses in Gy, and $\mathrm{M}$ is one month after end of therapy (when a total dose of 46 or 50 Gy was accumulated).

\begin{tabular}{cccccccc}
\hline Dose & 2 vs. 0 Gy & 10 vs. 2 Gy & 20 vs. 10 Gy & M vs. 20 Gy & 10 vs. 0 Gy & 20 vs. 0 Gy & M vs. 0 Gy \\
\hline Up-regulated miRNAs & 74 & 12 & 24 & 22 & 36 & 46 & 61 \\
Down-regulated miRNAs & 20 & 44 & 21 & 18 & 14 & 18 & 16 \\
\hline
\end{tabular}

The miRNAs from the training cohort were then grouped according to a significant change (up (U) or down (D), uncorrected $p$-value $<0.05$ ) or no change $(\mathrm{N})$ in the expression level between subsequent doses (higher versus lower) (Figure 2c). The largest branch (pattern group) contained no significantly altered miRNAs at any dose (380 miRNAs). The second largest branch displayed an upregulation of miRNAs by the first fraction and then no significant change with dose (51 miRNAs), while the third largest branch contained miRNAs which were downregulated at 10 Gy vs. 2 Gy, with no change in the other comparisons ( $22 \mathrm{miRNAs}$ ). Following the initial $\mathrm{N}$ response, there were both up- and downregulated miRNAs after $10 \mathrm{~Gy}$; however, after initial $\mathrm{U}$ response there were no upregulated miRNAs after $10 \mathrm{~Gy}$, and, after initial D response, there were no downregulated miRNAs after $10 \mathrm{~Gy}$. In none of the cases were significant changes in miRNA expression consistently correlated with the increasing or decreasing dose (UUUU or DDDD patterns).

\subsection{Dose Predictive miRNA Signatures}

In the next step, we searched for miRNA signatures which could predict the absorbed dose. A multinomial logistic regression with five possible outcomes representing dose points was used to build a statistical model with non-redundancy of the most discriminative miRNAs, relative to 0 Gy. Due to the small sample size, no additional feature selection method was used. After filtering (see Statistical Analysis section), 185 miRNAs were used to construct multinomial logistic regression models, and 1,055,425 multinomial logistic regression models (185 with 1 miRNA, 17,020 with 2 miRNAs, and 1,038,220 with 3 miRNAs) were built on 40 samples from the training dataset, and validated on the 15 samples lacking measurements for all doses (by-chance classification error rate equals $80.0 \%$ in training set and $78.2 \%$ in validation set). For each size of the miRNA signature $(n=1,2$, or 3$)$, the two best models were selected, as described in the Statistical Analysis section (Table S1; Figure S3).

The best single miRNA in the training set was miR-744-5p (training set error $=57.5 \%$; validation set error $=66.7 \%$ ), the best pair was miR-4461; miR-6513-5p (training set error $=42.5 \%$; validation set error $=73.3 \%$ ), and the best triplet was miR-103a-2-5p; miR1246; $\mathrm{miR}-454-3 \mathrm{p}$ (training set error $=30.0 \%$; validation set error $=73.3 \%$ ) (Figure 3a). For the best miRNAs in the validation set, the overlap was better: the best single was miR-34a-5p (training set error $=65.0 \%$; validation set error $=46.7 \%$ ), the best pair was miR-30b-3p; miR-34a-5p (training set error $=62.5 \%$; validation set error $=20.0 \%$ ), and the best triplet contained the miRNAs from the best pair plus miR-5690 (training set error $=50.0 \%$; validation set error $=20.0 \%$ ) (Figure $3 b$ ). Although the overall level of falsely identified miRNAs was relatively high (Table S1; Figure S3), the general patterns of the identified miRNAs displayed a clear concordance in upregulation or downregulation. However, at an individual level, a high level of variability was evident. 
a
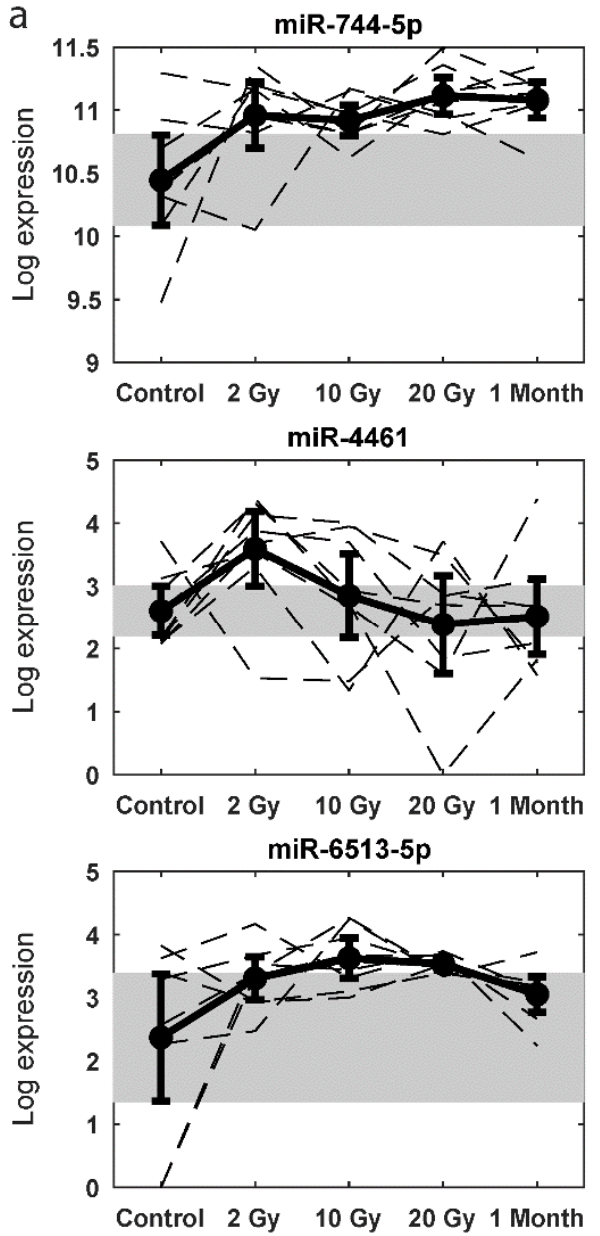
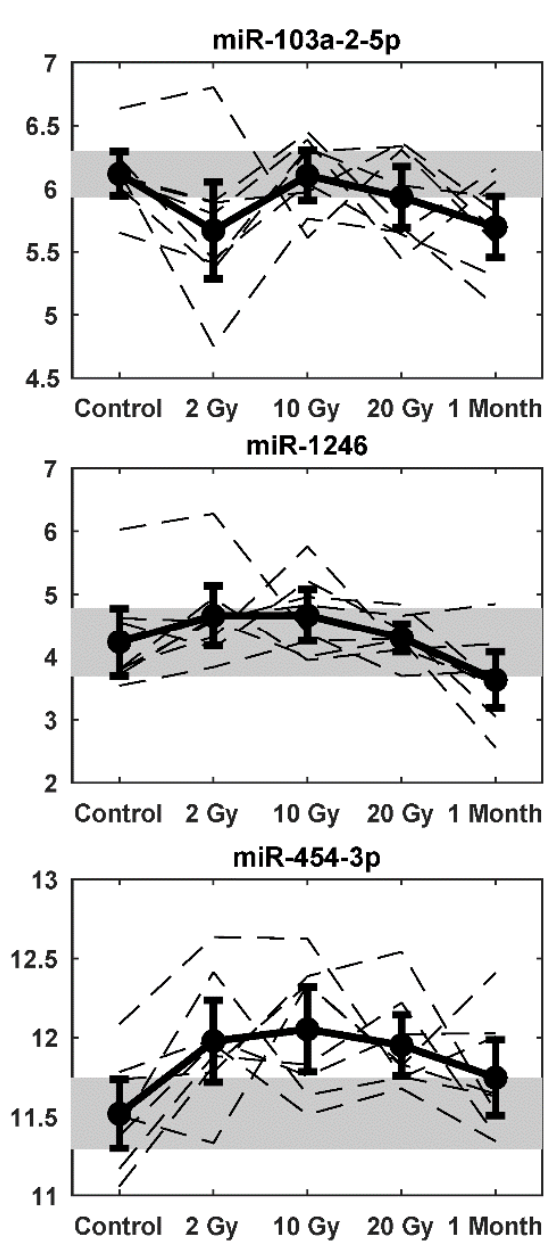

b
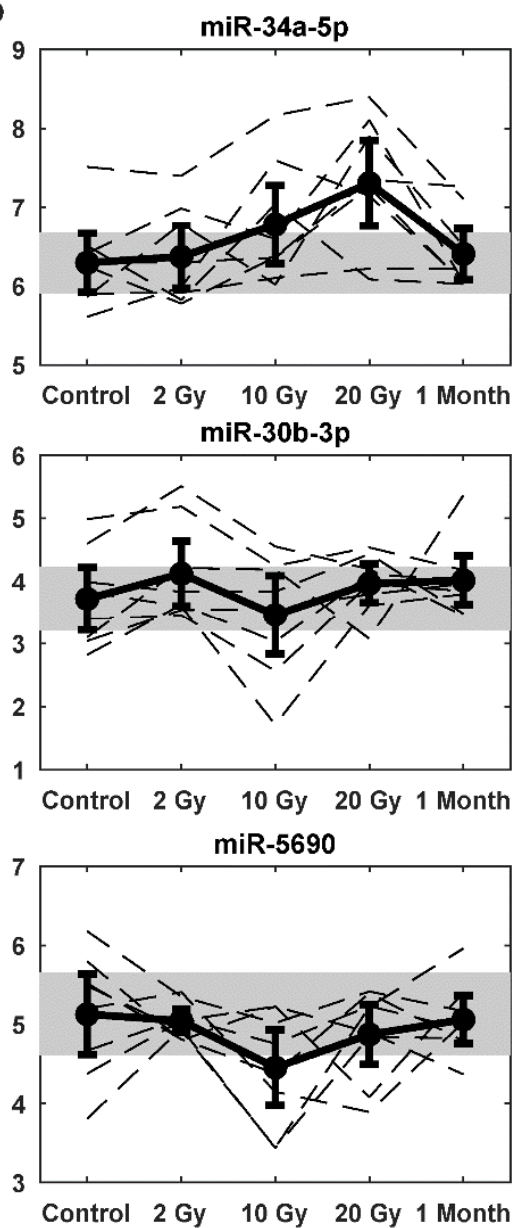

Figure 3. Selected target miRNAs based on model building from the training set (a) and the validation set (b). Dotted lines show expression values in individual patients, while the bold, solid line is the average expression across patients with $95 \%$ confidence intervals. Grey areas represent confidence intervals of average expression for the controls.

\subsection{Interacting, Dose Informative miRNAs}

With the available information on the expression patterns of all miRNAs, it was interesting to identify miRNA which showed the highest level of interaction with other miRNA as a function of dose. When comparing each dose to $0 \mathrm{~Gy}$ using the Broadside algorithm (Figure 4a), the highest main effects (the impact of individual miRNA to dose change) were estimated for miR-181a-2-3p and miR-181b-2-3p. The strongest interactions (added value of using two miRNAs together) were observed between miR-181b-2-3p, miR181a-2-3p, miR-873-5p, and miR-4461, and also between miR-1538 and miR-659-5p. In the analysis of adjacent doses (Figure $4 b$ ), the highest main effects were found for miR-181a-5p, miR-1246, and miR-7977. Two main miRNAs interacted with many miRNAs: namely, miR-1246 interacted with miR-4746-5p and miR-889-3p, and miR-181a-5p interacted with miR-146a-5p, miR-34a-5p, and miR-26a-1-3p. Both miR-1246 and miR-4461 were already discovered in the model building step, which supports the hypothesis that these miRNAs may be promising dose predictors when in cooperation with other miRNAs; however, analyses in larger cohorts are needed. 
a

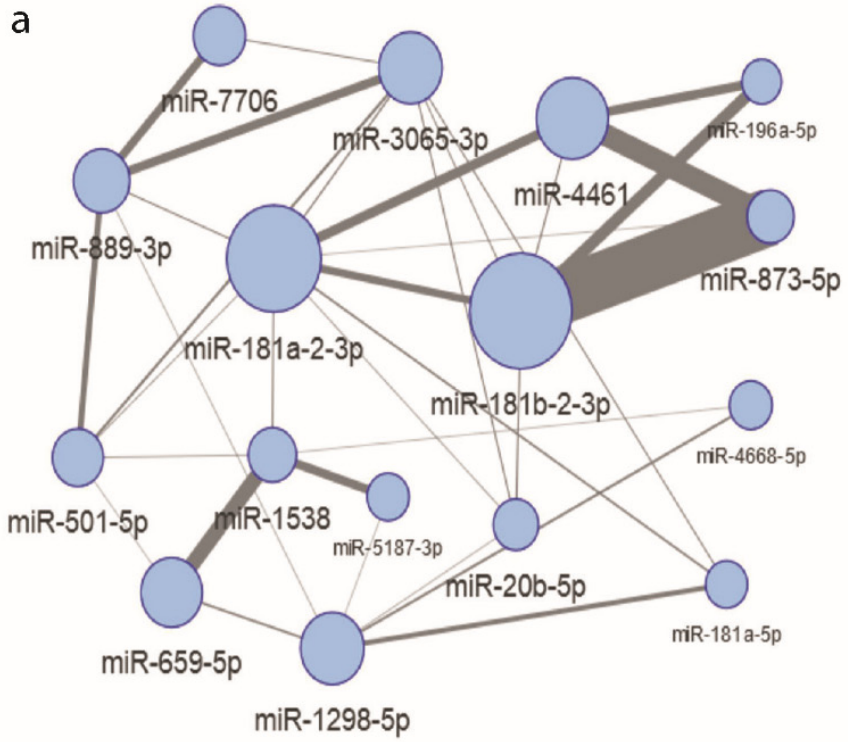

b

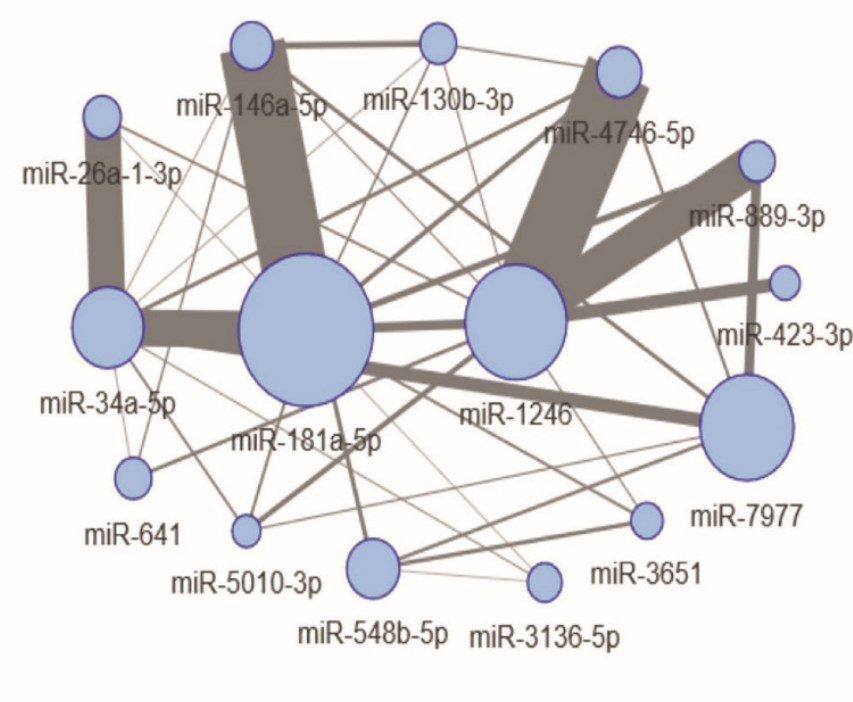

Figure 4. Graphs presenting dose informative interactions between miRNAs represented as circles for comparison of 0 Gy to other doses (a) and comparison of subsequent doses (b) using the Broadside algorithm. The diameter of a miRNA symbol represents the main effect (impact of the single miRNA on dose prediction), while the width of the line between miRNAs represents the strength of an interaction effect (impact of the pair of miRNAs on dose prediction).

\section{Discussion}

The aim of this investigation was to analyse the suitability of miRNAs in peripheral blood leukocytes as biomarkers of exposure and response to radiation. To this end, we analysed global miRNAs in leukocytes from breast cancer patients after they received total tumour doses of 2, 10, 20, or 48-50 Gy, given in 2 Gy fractions. The PCA plots indicate that there is a high level of intra- and inter-donor variability since few overall consistent patterns were visible using this method. Still, the average levels of several miRNA, alone or in combination, were recurrently modulated by radiation exposure.

Following multiple testing correction, only miR-3065-3p was found to be significantly decreased after $2 \mathrm{~Gy}$ with a trend towards a further decrease at higher doses. Although decreased in our study, previous reports indicated that miR-3065-3p is involved in DNA damage induction via the p53 signalling pathway, since it was upregulated by stable transfection of wild type p53 [15] and repressed when mutant p53 was stably transfected [16]. Although a $3 p$ strand miRNA is considered to play a minor role as compared with $5 p$, there are indeed numerous situations where the $3 p$ form is expressed and functional [17].

The most stably increased miRNA, miR-744-5p, was reported to be high in the plasma of pancreatic cancer patients and an independent marker of cancer progression and recurrence [18]. We cannot rule out the possibility that its increase reflects other tumour-related events, such as an enrichment of radiation resistant stem-like cells during therapy. Ex vivo experiments could shed more light on the relation between radiation-induced cellular damage and expression of this miRNA. miR-103a-2-5p showed, in combination with miR-1246 and miR-454-3p, the lowest classification error in both the training and validation sets. miR-103a-2-5p expression was elevated at 4,8 , and $24 \mathrm{~h}$ post $\mathrm{X}$-ray exposure, while it was decreased at $12 \mathrm{~h}$ after exposure in TK6 cells [19]. In our samples, the expression pattern was also sigmoidal but the timing was different. miR-103a-2-5p controls the expression level of the single strand break repair protein poly-(ADP-ribose) polymerase 1 (PARP1) and pre-miR-103a-2-5p transfection decreased PARP1 mRNA and enhanced DNA damage in primary human aortic endothelial cells [20]. Hence, its downregulation by radiotherapy is plausible and could possibly reflect a compensatory elevation of DNA damage response via PARP1. 
miR-1246 was a highly elevated exosomal miRNA at $24-48 \mathrm{~h}$ after irradiation in non-small cell lung cancer (NSCLC) cells [21] and is proposed to be a potential diagnostic serum biomarker for diverse cancers [21,22]. Our daily fractionation might have allowed miR-1246 to remain elevated at 2-10 Gy, although we measured intracellular levels. Tumour removal might have contributed to the minor decrease at 1 month post-therapy. The last triplet miRNA, miR-454-3p, targets the tumour suppressor gene B cell translocation gene 1 (BTG1), which is induced in response to stressors including X-rays [23]. Transfection of cells with miR-454-3p or BTG1 siRNA increased cellular radiosensitivity [23].

Model building on the validation set identified miR-34a-5p as the best candidate. Its gradual increase up to $20 \mathrm{~Gy}$ correlates with elevated levels in stimulated human $\mathrm{T}$ lymphocytes [24], as well as in total abdominal irradiated (TAI) mice, where effects were prevented by injecting an antagonistic miR-34a-5p [25]. miR-34a-5p is a well-known p53regulated tumour suppressor miRNA targeting RAD51, thereby inhibiting double strand break repair and sensitising to radiation [26]. The other two miRNAs identified were miR-30b-3p — weakly reduced after 10 Gy and also decreased at $12 \mathrm{~h}$ in TK6 cells [19]and miR-5690, with no previous connection to radiation response. As shown for these miRNAs, the temporal miRNA response has a role in the interpretation of the data. Due to the fractionation schedule, the dose increase is paralleled by an increase in time, but the response of certain miRNAs may be affected by time and not only dose. This is a factor to consider, since it is not possible to compare 2, 10, and 20 Gy of acute irradiation to each other using this setup.

What limits the use of radiation-modulated miRNAs as biomarkers of radiation exposure is the large individual variability. Generally, high variability in control miRNA levels restricts the creation of a calibration curve which could be used for assessing an absorbed dose in a person whose background level is not known. Such a strategy is possible with cytogenetic biomarkers of radiation exposure, because the background level of chromosomal damage is very low [27]. It is likely that the strong individual variability is related to the partial body exposure of radiotherapy patients. Similar observations were made for chromosomal aberrations and micronuclei in PBL of radiotherapy patients exposed to a single dose of radiation [28], as well as for gamma H2AX foci after fractionated irradiation [29], and is obviously related to the relatively low and variable fraction of lymphocytes which are in the radiation field when a dose is delivered [30]. Breast cancer patients were selected for this study largely due to the availability of samples, yet this choice may in retrospect have been a limitation of the study. It has lately become evident that this tumour type receives a lower calculated mean dose to blood cells than a number of other tumour types, and it also, therefore, has a relatively low increase in expression of the radiation-responsive gene FDXR per dose given externally at $24 \mathrm{~h}$ after the first fraction [31]. It is logical that irradiated blood volume has been suggested as an important determinant for the radiation-induced transcriptional response when comparing tissues [31,32], and since the irradiated tumour volume is not constant, this creates variability between patients. Thus, high individual variability does not preclude the possibility that a more homogeneous response is achieved after whole body exposure. Indeed, reproducible dose-response relationships were obtained with mRNA measurements in lymphocytes of cancer patients receiving total body irradiation [33]. It is also possible that serum samples, containing secreted miRNA from the irradiated tissue, can represent the irradiated tissue better than leukocytes in the case of partial body irradiation. Finally, the number of patients in this study posed a limitation as well, although it would have been less of a problem if the baseline levels were as stable as when using the chromosomal aberration assay. Additional patients could not easily be included in this study later, however, due to altered fractionation schemes for breast cancer radiotherapy at the nearby hospital.

In conclusion, our study reports on several potentially radiation-induced miRNAs, in particular the increase in miR-744-5p. Additionally, this type of analysis of samples from radiotherapy patients further suggests that the irradiated blood volume may be an important factor when analysing leukocytes. 


\section{Materials and Methods}

\subsection{Patient Samples}

Blood samples were drawn from 16 patients with breast cancer undergoing radiotherapy (details in [12]). Ten millilitres of blood was sampled before radiotherapy, on the day when $1 \times 2$ Gy (100\% prescription isodose to the tumour), $5 \times 2$ (10) Gy, $10 \times 2$ (20) Gy was reached, and one month after 23 or 25 fractions of 2 Gy (" 1 month", 46 or 50 Gy from 23 or $25 \times 2 \mathrm{~Gy}$, respectively). Reasons for incomplete data were that the cell pellets were limited for some samples and did not yield RNA of sufficient quantity or quality. The ethical approval was performed by the regional ethical review board (no. 2016/1361-32, which is an amendment to 2010/1726-31/4). The early side effects to radiotherapy of these patients were classified as grade 1-2 using the Radiation Therapy Oncology Group scale.

\subsection{Sample Preparation}

Blood was collected in heparinised tubes and kept on ice for at least $10 \mathrm{~min}$. Leukocytes were separated from red blood cells by gentle mixing of blood with red blood cell lysis buffer (RCLB, containing $0.15 \mathrm{M} \mathrm{NH}_{4} \mathrm{Cl}, 10 \mathrm{mM} \mathrm{KHCO}_{3}$, and $0.1 \mathrm{mM}$ EDTA, set to $\mathrm{pH} 7.3$ ) for $20-60 \mathrm{~min}$ at $4{ }^{\circ} \mathrm{C}$. After centrifugation at $300 \times \mathrm{g}$ at $4{ }^{\circ} \mathrm{C}$ for $5 \mathrm{~min}$, cells were washed with RCLB again, then with phosphate-buffered saline (PBS). The prepared leukocytes were frozen at $-150{ }^{\circ} \mathrm{C}$ in RPMI medium supplemented with $10 \%$ defined bovine serum (DBS), 1\% PEST, and 10\% DMSO (all from Sigma-Aldrich, Stockholm, Sweden). Before RNA preparation, samples were thawed quickly using a $37^{\circ} \mathrm{C}$ water bath, cells were pelleted at $1500 \mathrm{rpm}$ for $4 \mathrm{~min}$, and washed with PBS.

The miRNeasy Mini Kit (Qiagen, Sollentuna, Sweden) was used for RNA preparation, combining phenol/guanidine-based lysis of samples with silica membrane purification of total RNA from 18 nucleotides and upwards. RNA quality was assessed using an Agilent 2100 Bioanalyzer with an Agilent RNA 6000 Nano Kit (2100 Expert Eukaryote Total RNA Nano, Agilent Technologies Sweden AB, Kista, Sweden), where an RNA integrity number (RIN) $>5$ is regarded as good quality, and $>8$ as perfect [34]. Our samples displayed RIN of $5.9 \pm 1.6$ (average \pm standard deviation), however the value was not available for $18 / 52$ samples (35\%). Upon receipt at the National Genomics Infrastructure Sweden, RIN values of $4.5 \pm 0.5$ were given using the Qubit Fluorometer. We decided to proceed with the samples, despite the relatively low RIN values, since RIN values were reported to have negligible or no effect on miRNA analysis, while accuracy is more commonly reduced for mRNA analysis [35].

\subsection{RNA Sequencing and Reads Pre-Processing}

Library preparation was carried out by a modified version of the Illumina TruSeq Small RNA library preparation protocol. Sequencing was performed on the Illumina HiSeq 2500 platform with high output mode, V4 reagents, and $1 \times 50$ single-end setup. Raw sequence reads were trimmed with trim_galore v. 0.4.5. [36]. Sequence reads shorter than $18 \mathrm{bp}$ after trimming or Phred quality score lower than 20 were removed from downstream analysis. Alignment was performed against human mature miRNA sequences in miRBase v21 containing, in total, 35,828 mature miRNA products in 223 species, where 2588 mature miRNAs were human. For this purpose, the QuickMIRSeq algorithm was used, which incorporates the strand information in the alignment, filters out reads potentially arising from background noise, and remaps sequences aligned to miRNAs with mismatches to a reference genome to further reduce false positives [37]. The application of QuickMIRSeq with filtering and remapping of mismatches gave quantified expression for 879 miRNAs.

\subsection{Data Cleaning and Imputation}

In the first step, for each miRNA and radiation dose of 5 patients with complete information (patients 7, 9, 14-16, Table 1), outlier expression values were detected using Dixon's Q-test. For each dose separately, detected outlier values were replaced with the corresponding values from the nearest-neighbour patient. Patients' similarity was 
estimated using correlation-based measures. In the second step, cleaned expression data for 5 patients were used to impute measurements for 3 patients with only one single dose missing (patients $8,12,13$, Table 1). For each miRNA and patient, a missing dose measurement was imputed with the corresponding value from the nearest-neighbour patient from the complete 5-patient set. Imputed values did not change the distribution of the miRNA's expression level. In this step, 3 from 40 samples $(7.5 \%)$ were imputed.

\subsection{Statistics of Sequencing Data}

In total, 20-50 million sequence reads were obtained for all samples, and $80-95 \%$ of them were retained after adapter trimming and quality filtering. The mean insert sizes were $25-35 \mathrm{bp}$, which is slightly longer than the theoretical length of miRNA (20-22 bp). This is acceptable since, during library preparation, other types of small RNAs are also selected. For all samples $90-97 \%$ of filtered reads could be aligned to the human reference genome GRCh37. For most of the samples, $10-30 \%$ of reads were aligned to human mature miRNA in miRbase [38], and 10-20\% of reads aligned to human miRNA precursors. Since we aligned reads to miRBase, only miRNA expression was analysed in this study.

\subsection{Filtering and Normalisation}

Low-expression miRNAs were filtered using the threshold of a minimum of 5 counts in at least $50 \%$ of the samples, as suggested in [39]. In the training set, 306 miRNAs with low expression were removed (573 miRNAs left). In the validation group, the same miRNAs were removed. Normalization was performed using the upper quartile (UQ) method. It scales the expression data using the third quantile of expression values for each sample separately. Finally, data were transformed using $\log 2(x+1)$ to reduce the skewness and the number of extreme values.

\subsection{Statistical Analysis}

A $t$-test for paired samples was used to find differences in miRNA expression between subsequent doses. Left-tail and right-tail hypotheses were investigated separately to estimate the direction of expression change between doses. The Benjamini-Hochberg algorithm was used for multiple testing correction by controlling the false discovery rate. Due to small sample sizes in this study, miRNA was treated as differentially expressed if the $p$-value, without correction for multiple testing, was smaller than 0.05 .

Prior to model building, miRNAs with $p$-values from univariate analysis higher than 0.05 in all comparisons between each dose and no radiation ( $0 \mathrm{~Gy})$ were filtered away (185 miRNAs left). A multinomial logistic regression with 5 possible outcomes representing dose points (relative to $0 \mathrm{~Gy}$ ) was used to build a statistical model on the training set, which was then tested on the validation set. No additional feature selection method was used, except the feature filtering described above. Instead, the best combinations of 1,2, and 3 miRNAs were found, giving miRNA signatures of dose prediction. For each size of the signature, we selected two final models assuming the following scenarios: (1) from the pool of models with the lowest classification errors in the training set, we chose the one with the lowest classification error in the validation set, and tagged the selected signature as the best training set miRNAs; (2) from the pool of models with the lowest classification errors in the validation data, we chose the one with the lowest classification error in the training data, and tagged the selected signature as best validation set miRNAs. The by-chance classification error rate was computed as a sum of squared proportions of samples classified to each dose point.

An explorative feature selection and interaction mining tool called Broadside (downloaded from http:/ / zaed.aei.polsl.pl/index.php/pl/oprogramowanie-zaed on 10 November 2017) was used to discover sets of interacting informative miRNAs [40]. Broadside consists of a series of miRNA permutations, combined with a flexible decomposition of the miRNA total effect into main and interaction effects. Main effect measures the impact of individual miRNA on the outcome (dose change), and interaction effect represents the 
added value of using two miRNAs together. To preserve the paired nature of the data, two analysis scenarios were introduced: (1) expression differences were calculated between any dose and $0 \mathrm{~Gy}$; (2) expression differences were calculated between adjacent doses (0 vs. 2 Gy, 2 vs. 10 Gy, 10 vs. 20 Gy, and 20 Gy vs. 1 month).

Supplementary Materials: The following are available online at https:/ /www.mdpi.com/article/10 $.3390 / \mathrm{ijms} 22168705 / \mathrm{s} 1$.

Author Contributions: Conceptualization, A.W. and L.L.; methodology, A.W. and L.L.; software, M.M. and J.P.; validation, M.M. and L.L.; formal analysis, M.M.; investigation, L.L.; resources, J.P., A.W., and L.L.; data curation, M.M.; writing-original draft preparation, L.L., M.M., and A.W.; writing-review and editing, all authors; visualization, M.M.; supervision, J.P. and A.W.; project administration, M.M. and L.L.; funding acquisition, J.P., A.W., and L.L. All authors have read and agreed to the published version of the manuscript.

Funding: This research was funded by the European Union's Seventh Framework Programme (FP7/2007-2013), grant number 241,536, and Stockholm University, grant number SU FV-5.1.23222-14. M.M. and J.P. were co-financed by the Silesian University of Technology, grant number BK-218/RAU4/2021 and L.L. was co-financed by Stiftelsen Olle Engkvist Byggmästare, grant number 184-510. The authors acknowledge support from Science for Life Laboratory, the Knut and Alice Wallenberg Foundation, the National Genomics Infrastructure funded by the Swedish Research Council, and the Uppsala Multidisciplinary Center for Advanced Computational Science for their assistance with massively parallel sequencing and access to the UPPMAX computational infrastructure.

Institutional Review Board Statement: The study was conducted according to the guidelines of the Declaration of Helsinki, and approved by the regional ethical review board in Stockholm (protocol code 2016/1361-32, date of approval 7 July 2016).

Informed Consent Statement: Informed consent was obtained from all subjects involved in the study.

Data Availability Statement: The data sets supporting the results of this article are available in the NCBI Sequence Read Archive (SRA) repository under project no. PRJNA531002.

Conflicts of Interest: The authors declare no conflict of interest. The funders had no role in the design of the study; in the collection, analyses, or interpretation of data; in the writing of the manuscript; or in the decision to publish the results.

\section{References}

1. ICRP. Prevention of Accidental Exposures to Patients Undergoing Radiation Therapy; International Commission on Radiological Protection: Ottawa, ON, Canada, 2001.

2. Dorr, W. Radiobiology of tissue reactions. Ann. ICRP 2015, 44, 58-68. [CrossRef]

3. Newhauser, W.D.; Berrington de Gonzalez, A.; Schulte, R.; Lee, C. A Review of Radiotherapy-Induced Late Effects Research after Advanced Technology Treatments. Front. Oncol 2016, 6, 13. [CrossRef] [PubMed]

4. Bentzen, S.M. Potential clinical impact of normal-tissue intrinsic radiosensitivity testing. Radiother. Oncol 1997, 43, 121-131. [CrossRef]

5. Averbeck, D.; Candeias, S.; Chandna, S.; Foray, N.; Friedl, A.A.; Haghdoost, S.; Jeggo, P.A.; Lumniczky, K.; Paris, F.; Quintens, R.; et al. Establishing mechanisms affecting the individual response to ionizing radiation. Int. J. Radiat. Biol. 2020, 96, 297-323. [CrossRef]

6. Bergom, C.; West, C.M.; Higginson, D.S.; Abazeed, M.E.; Arun, B.; Bentzen, S.M.; Bernstein, J.L.; Evans, J.D.; Gerber, N.K.; Kerns, S.L.; et al. The Implications of Genetic Testing on Radiation Therapy Decisions: A Guide for Radiation Oncologists. Int. J. Radiat. Oncol. Biol. Phys. 2019, 105, 698-712. [CrossRef] [PubMed]

7. Prasanna, P.G.S.; Narayanan, D.; Zhang, K.; Rahbar, A.; Coleman, C.N.; Vikram, B. Radiation Biomarkers: Can Small Businesses Drive Accurate Radiation Precision Medicine? Radiat. Res. 2020, 193, 199-208. [CrossRef] [PubMed]

8. Rajaraman, P.; Hauptmann, M.; Bouffler, S.; Wojcik, A. Human individual radiation sensitivity and prospects for prediction. Ann. ICRP 2018, 47, 126-141. [CrossRef]

9. Malicki, J. The importance of accurate treatment planning, delivery, and dose verification. Rep. Pract. Oncol. Radiother. J. Greatpoland Cancer Cent. Pozn. Pol. Soc. Radiat. Oncol. 2012, 17, 63-65. [CrossRef]

10. Mijnheer, B.; Beddar, S.; Izewska, J.; Reft, C. In vivo dosimetry in external beam radiotherapy. Med. Phys. 2013, 40, 070903. [CrossRef]

11. Tanderup, K.; Beddar, S.; Andersen, C.E.; Kertzscher, G.; Cygler, J.E. In vivo dosimetry in brachytherapy. Med. Phys. 2013, 40, 070902. [CrossRef] 
12. Deperas-Kaminska, M.; Bajinskis, A.; Marczyk, M.; Polanska, J.; Wersäll, P.; Lidbrink, E.; Ainsbury, E.A.; Guipaud, O.; Bender, M.A.; Haghdoost, S.; et al. Radiation induced changes in levels of selected proteins in peripheral blood serum of breast cancer patients as a potential triage biodosimeter for large-scale radiological emergencies. Health Phys. 2014, 107, 555-563. [CrossRef]

13. Jacob, N.K.; Cooley, J.V.; Yee, T.N.; Jacob, J.; Alder, H.; Wickramasinghe, P.; Maclean, K.H.; Chakravarti, A. Identification of sensitive serum microRNA biomarkers for radiation biodosimetry. PLoS ONE 2013, 8, e57603. [CrossRef] [PubMed]

14. Wei, W.; He, J.; Wang, J.; Ding, N.; Wang, B.; Lin, S.; Zhang, X.; Hua, J.; Li, H.; Hu, B. Serum microRNAs as Early Indicators for Estimation of Exposure Degree in Response to Ionizing Irradiation. Radiat. Res. 2017, 188, 342-354. [CrossRef]

15. Hunten, S.; Kaller, M.; Drepper, F.; Oeljeklaus, S.; Bonfert, T.; Erhard, F.; Dueck, A.; Eichner, N.; Friedel, C.C.; Meister, G.; et al. p53-Regulated Networks of Protein, mRNA, miRNA, and lncRNA Expression Revealed by Integrated Pulsed Stable Isotope Labeling With Amino Acids in Cell Culture (pSILAC) and Next Generation Sequencing (NGS) Analyses. Mol. Cell. Proteom. MCP 2015, 14, 2609-2629. [CrossRef] [PubMed]

16. Zhang, Y.; Hu, Y.; Fang, J.Y.; Xu, J. Gain-of-function miRNA signature by mutant p53 associates with poor cancer outcome. Oncotarget 2016, 7, 11056-11066. [CrossRef] [PubMed]

17. Desvignes, T.; Batzel, P.; Berezikov, E.; Eilbeck, K.; Eppig, J.T.; McAndrews, M.S.; Singer, A.; Postlethwait, J.H. miRNA Nomenclature: A View Incorporating Genetic Origins, Biosynthetic Pathways, and Sequence Variants. Trends Genet. TIG 2015, 31, 613-626. [CrossRef]

18. Miyamae, M.; Komatsu, S.; Ichikawa, D.; Kawaguchi, T.; Hirajima, S.; Okajima, W.; Ohashi, T.; Imamura, T.; Konishi, H.; Shiozaki, A.; et al. Plasma microRNA profiles: Identification of miR-744 as a novel diagnostic and prognostic biomarker in pancreatic cancer. Br. J. Cancer 2015, 113, 1467-1476. [CrossRef]

19. Chaudhry, M.A.; Omaruddin, R.A.; Brumbaugh, C.D.; Tariq, M.A.; Pourmand, N. Identification of radiation-induced microRNA transcriptome by next-generation massively parallel sequencing. J. Radiat. Res. 2013, 54, 808-822. [CrossRef]

20. Dluzen, D.F.; Kim, Y.; Bastian, P.; Zhang, Y.; Lehrmann, E.; Becker, K.G.; Noren Hooten, N.; Evans, M.K. MicroRNAs Modulate Oxidative Stress in Hypertension through PARP-1 Regulation. Oxid. Med. Cell. Longev. 2017, 2017, 3984280. [CrossRef]

21. Yuan, D.; Xu, J.; Wang, J.; Pan, Y.; Fu, J.; Bai, Y.; Zhang, J.; Shao, C. Extracellular miR-1246 promotes lung cancer cell proliferation and enhances radioresistance by directly targeting DR5. Oncotarget 2016, 7, 32707-32722. [CrossRef]

22. Sakha, S.; Muramatsu, T.; Ueda, K.; Inazawa, J. Exosomal microRNA miR-1246 induces cell motility and invasion through the regulation of DENND2D in oral squamous cell carcinoma. Sci. Rep. 2016, 6, 38750. [CrossRef]

23. Wu, X.; Ding, N.; Hu, W.; He, J.; Xu, S.; Pei, H.; Hua, J.; Zhou, G.; Wang, J. Down-regulation of BTG1 by miR-454-3p enhances cellular radiosensitivity in renal carcinoma cells. Radiat. Oncol. 2014, 9, 179. [CrossRef]

24. Kabacik, S.; Manning, G.; Raffy, C.; Bouffler, S.; Badie, C. Time, dose and ataxia telangiectasia mutated (ATM) status dependency of coding and noncoding RNA expression after ionizing radiation exposure. Radiat. Res. 2015, 183, 325-337. [CrossRef]

25. Cui, M.; Xiao, H.; Li, Y.; Dong, J.; Luo, D.; Li, H.; Feng, G.; Wang, H.; Fan, S. Total abdominal irradiation exposure impairs cognitive function involving miR-34a-5p/BDNF axis. Biochim. Biophys. Acta 2017, 1863, 2333-2341. [CrossRef]

26. Cortez, M.A.; Valdecanas, D.; Niknam, S.; Peltier, H.J.; Diao, L.; Giri, U.; Komaki, R.; Calin, G.A.; Gomez, D.R.; Chang, J.Y.; et al. In Vivo Delivery of miR-34a Sensitizes Lung Tumors to Radiation Through RAD51 Regulation. Mol. Ther. Nucleic Acids 2015, 4, e270. [CrossRef] [PubMed]

27. IAEA. Cytogenetic Dosimetry: Applications in Preparedness for and Response to Radiation Emergencies; International Energy Agency: Vienna, Austria, 2011.

28. Wojcik, A.; Stephan, G.; Sommer, S.; Buraczewska, I.; Kuszewski, T.; Wieczorek, A.; Gozdz, S. Chromosomal aberrations and micronuclei in lymphocytes of breast cancer patients after an accident during radiotherapy with $8 \mathrm{MeV}$ electrons. Radiat. Res. 2003, 160, 677-683. [CrossRef] [PubMed]

29. Sak, A.; Grehl, S.; Erichsen, P.; Engelhard, M.; Grannass, A.; Levegrun, S.; Pottgen, C.; Groneberg, M.; Stuschke, M. gammaH2AX foci formation in peripheral blood lymphocytes of tumor patients after local radiotherapy to different sites of the body: Dependence on the dose-distribution, irradiated site and time from start of treatment. Int. J. Radiat. Biol. 2007, 83, 639-652. [CrossRef] [PubMed]

30. D'Alesio, V.; Pacelli, R.; Durante, M.; Canale Cama, G.; Cella, L.; Gialanella, G.; Grossi, G.; Pugliese, M.; Punzo, G.; Sardi, I.; et al . Lymph nodes in the irradiated field influence the yield of radiation-induced chromosomal aberrations in lymphocytes from breast cancer patients. Int. J. Radiat. Oncol. Biol. Phys. 2003, 57, 732-738. [CrossRef]

31. O’Brien, G.; Cruz-Garcia, L.; Majewski, M.; Grepl, J.; Abend, M.; Port, M.; Tichy, A.; Sirak, I.; Malkova, A.; Donovan, E.; et al. FDXR is a biomarker of radiation exposure in vivo. Sci. Rep. 2018, 8, 684. [CrossRef] [PubMed]

32. Manning, G.; Tichy, A.; Sirak, I.; Badie, C. Radiotherapy-Associated Long-term Modification of Expression of the Inflammatory Biomarker Genes ARG1, BCL2L1, and MYC. Front. Immunol. 2017, 8, 412. [CrossRef]

33. Amundson, S.A.; Grace, M.B.; McLeland, C.B.; Epperly, M.W.; Yeager, A.; Zhan, Q.; Greenberger, J.S.; Fornace, A.J., Jr. Human in vivo radiation-induced biomarkers: Gene expression changes in radiotherapy patients. Cancer Res. 2004, 64, 6368-6371. [CrossRef] [PubMed]

34. Fleige, S.; Pfaffl, M.W. RNA integrity and the effect on the real-time qRT-PCR performance. Mol. Asp. Med. 2006, 27, 126-139. [CrossRef] [PubMed]

35. Jung, M.; Schaefer, A.; Steiner, I.; Kempkensteffen, C.; Stephan, C.; Erbersdobler, A.; Jung, K. Robust microRNA stability in degraded RNA preparations from human tissue and cell samples. Clin. Chem. 2010, 56, 998-1006. [CrossRef] [PubMed] 
36. Trim_Galore. Core Script: Trim_Galore-Small_Rna \$RAW.Fastq.gz-Length 18-Gzip -o \$TRIMMED.Fastq.gz. Available online: http://www.bioinformatics.babraham.ac.uk/projects/trim_galore/ (accessed on 12 October 2016).

37. Zhao, S.; Gordon, W.; Du, S.; Zhang, C.; He, W.; Xi, L.; Mathur, S.; Agostino, M.; Paradis, T.; von Schack, D.; et al. QuickMIRSeq: A pipeline for quick and accurate quantification of both known miRNAs and isomiRs by jointly processing multiple samples from microRNA sequencing. BMC Bioinform. 2017, 18, 180. [CrossRef] [PubMed]

38. Kozomara, A.; Birgaoanu, M.; Griffiths-Jones, S. miRBase: From microRNA sequences to function. Nucleic Acids Res. 2019, 47, D155-D162. [CrossRef]

39. Tam, S.; Tsao, M.S.; McPherson, J.D. Optimization of miRNA-seq data preprocessing. Brief. Bioinform. 2015, 16, 950-963. [CrossRef]

40. Krol, L.; Polanska, J. Multidimensional Feature Selection and Interaction Mining with Decision Tree Based Ensemble Methods; Springer: Cham, Switzerlnad, 2017; pp. 118-125. 